
Terminological Series issued by the Foreign Office of the Federal Republic of Germany Volume 3

Terminologische Schriftenreihe herausgegeben vom Auswärtigen Amt der Bundesrepublik Deutschland Band 3

Série de publications terminologiques du Ministère des Affaires étrangères de la République fédérale d'Allemagne tome 3

Publicaciones terminológicas

editadas por el Ministerio de Relaciones Exteriores de la República Federal de Alemania Volumen 3

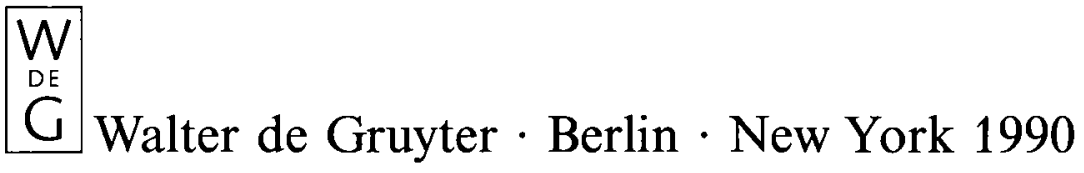




\section{German Institutions}

Designations, Abbreviations, Acronyms

compiled by the Language Services Division

of the Foreign Office of the Federal Republic of Germany

\section{Deutsche Einrichtungen}

Bezeichnungen, Abkürzungen, Akronyme

zusammengestellt vom Sprachendienst des Auswärtigen Amts

der Bundesrepublik Deutschland

\section{Institutions allemandes}

Dénominations, abréviations, acronymes

répertoire établi par les Services linguistiques

du Ministère des Affaires étrangères

de la République fédérale d'Allemagne

\section{Instituciones Alemanas}

Denominaciones, abreviaturas y acrónimos

compilados por el Servicio de Idiomas

del Ministerio de Relaciones Exteriores

de la República Federal de Alemania 


\section{Library of Congress Cataloging-in-Publication Data}

German institutions : designations, abbreviations, acronyms / compiled by the Language Services Division of the Foreign Office of the Federal Republic of Germany $=$ Deutsche Einrichtungen :

Zusammengestellt vom Sprachendienst des Auswärtigen Amts der Bundesrepublik Deutschland.

p. $\quad \mathrm{cm}-($ Terminological series $\mathrm{v} \cdot 3=$

Terminologische Schriftenreihe : Bd. 3)

English, German, French, and Spanish.

Includes index.

ISBN 0-89925-584-1 : DM 48.00 (\$29.00 : U.S.)

1. Associations, institutions, etc. - Germany (West) Directories. 2. Associations, institutions, etc. Germany (West) - Abbreviations-Dictionaries.

I. Germany (West). Auswärtiges Amt. Sprachendienst.

II. Title: Deutsche Einrichtungen.

III. Series: Terminological series ; v. 3 .

AS178.G47 1989

$063-\mathrm{dc} 20$

\section{CIP-Titelaufnahme der Deutschen Bibliothek}

German institutions : designations, abbreviations, acronyms $=$ Deutsche Einrichtungen $:$ Bezeichnungen, Abkürzungen, Akronyme = Institutions allemandes / comp. by the Language Services Division of the Foreign Office of the Federal Republic of Germany. Berlin ; New York : de Gruyter, 1989

(Terminological series ; Vol. 3)

ISBN 3-11-012087-9

NE: Deutschland < Bundesrepublik > / Auswärtiges

Amt /

Sprachendienst; 1. PT; GT

(C) Copyright 1989 by Walter de Gruyter \& Co., 1000 Berlin 30.

All rights reserved, including those of translation into foreign languages. No part of this book may be reproduced in any form - by photoprint, microfilm, or any other means - nor transmitted nor translated into a machine language without written permission from the publisher.

Printing: Ernst Kieser GmbH, Graphischer Betrieb, Augsburg.

Binding: Verlagsbuchbinderei Dieter Mikolai, Berlin.

Printed in Germany. 